\title{
Estrategia de intervención educativa sobre la salud bucal en pacientes de 6 a 32 años con Síndrome de Down
}

\author{
Educational intervention strategy oral health in patients from 6 to 32 years with Down Syndrome \\ Jackelym Aguirre Córdova 1,a, Diandra Porras Rodríguez 2,b, Katty Ríos Villasis 3,c,d,e
}

\section{RESUMEN}

Objetivos: Diseñar, implementar y evaluar la aplicación de juegos colectivos adaptados a pacientes de 6 a 32 años con Síndrome de Down, como estrategia de intervención educativa en la Salud Bucal. Material y métodos: Se realizó un estudio comparativo, observacional, longitudinal y prospectivo; la muestra estuvo conformada por 50 pacientes de 6 a 32 años del "Instituto De Rehabilitación Infantil y Educación Especial Milagroso Niño De Jesús” ubicado en la ciudad de Lima. Se diseñaron cuatro juegos colectivos con componente didáctico de acuerdo con el diagnóstico psico-pedagógico, los aportes de los docentes y la prueba piloto previa a la ejecución del estudio. Se implementaron y ejecutaron los juegos en el ámbito escolar en un consultorio de la parte médica, sin interrumpir las actividades diarias; asistieron por grupos de acuerdo a edad y aula, donde se explicó en forma dinámica los juegos e importancia de cada uno. Resultados: Se encontró una diferencia significativa entre las medias del Índice de Higiene Oral antes y después de la intervención educativa $(p<0,05)$. Conclusiones: La estrategia de intervención educativa disminuye considerablemente el índice de higiene oral en pacientes con sindrome de down.

PALABRAS CLAVE: Síndrome de Down, estrategia educativa, salud oral.

\section{SUMMARY}

Objectives: Design, implement and evaluate the application of collective v games adapted to Patients 6 to 32 years old with Down syndrome, as a strategy for Educational Intervention in Oral Health. Material and Methods: A comparative study, observational, longitudinal and prospective study was conducted; was the sample consisted of 50 patients aged 6 to 32 years of "Children's Rehabilitation Institute and Special Education Child Of Jesus Miracle" located in the city of Lima. Four collective games with educational component according to 
Aguirre-Córdova J, Porras-Rodríguez D, Ríos Villasis K.

the psycho-pedagogical diagnosis, the contributions of the teachers and the pilot prior to the execution of the study was designed. They were implemented and executed the games in schools in an office of the medical side without interrupting daily activities. They attended by Age Group and a classroom Agreement, which was explained as Dynamics and Importance games each one. Results: A significant difference between the averages of the index Oral hygiene before and after the educational intervention $(\mathrm{p}<0.05)$ was found. Conclusions: The oral hygiene index decreased significantly after the educational intervention strategy.

\section{KEYWORDS: Down syndrome, educational strategy, oral health}

\section{INTRODUCCIÓN}

La enfermedad genética más común de la especie humana es el síndrome de Down. El niño con síndrome de Down es un individuo único y diferente, sus alegrías y gustos son especiales, lo mismo que su desarrollo físico y mental, el cual está determinado tanto por su alteración genética como por sus características (1).

La causa del síndrome de Down es una alteración cromosómica, la presencia de 3 cromosomas 21, en vez de un par, que es lo normal. Este cromosoma 21 extra puede verse en el cariotipo en cualquiera de las tres formas que se denominan: TRISOMIA LIBRE, causante de la gran mayoría de los casos del S. Down; TRANSLOCACION, en estos casos la anomalía puede haber sido trasmitida de alguno de los padres y es necesario hacer el estudio cromosómico de ellos para evaluar el riesgo de recurrencia, y MOSAICISMO, en la que el niño con S. Down tiene una mezcla de células normales y otras con trisomía. Aunque el cuadro clínico es el mismo en uno y otro caso, cada una de las formas tiene diferente riesgo de recurrencia en la familia (2).

Este síndrome se presenta con mayor frecuencia en el varón. Existe una mayor incidencia de esta alteración a medida que aumenta la edad de la madre. Las características conductuales de estos pacientes incluyen espontaneidad, calidez, paciencia, una disposición amigable y casi siempre bondadosa (3) ${ }^{3}$ Aunque estas personas tienen derecho a las mismas normas de salud que la población general, es evidente el mayor número de problemas orales, relacionados con la mayor dificultad que presentan para obtener un cuidado adecuado de su higiene oral $(4,5)$.

Las personas con Síndrome de Down describen cambios y manifestaciones clínicas orales frecuentes como: pigmentaciones, gingivitis crónica generaliza- da, cálculos, enfermedad periodontal severa, pérdida ósea y pérdida prematura de dientes, aumentando su incidencia y severidad por el difícil acceso a los servicios de salud de este grupo de personas (6-10).

La enfermedad periodontal es la más común de las patologías bucales asociadas con este síndrome. En la actualidad es evidente que las personas con Síndrome de Down tienen una mayor susceptibilidad a contraer enfermedad periodontal, sin embargo no es clara la razón de ello (11).

Para el abordaje de la atención odontológica de las personas con necesidades especiales es preciso mejorar su higiene bucal por medio de programas educativos dirigidos al personal de atención y a las familias que ayudan con las tareas diarias del cuidado personal, establecer una formación académica y entrenamiento específico para profesionales de la odontología y asignar fondos para cubrir el acceso a los servicios de salud de este tipo de pacientes (12).

Para lograr la participación y la independencia de algunas personas con necesidades especiales en la realización de su higiene oral, resulta muy beneficiosa la utilización de estrategias de intervención educativa. Deben ser realizadas de preferencia con fotografías, ya que la fotografía es lo que más se asemeja a la imagen real de la boca y de los implementos con que se realiza la higiene oral, también los juegos musicales son muy bien recibidos por el paciente. Estos recursos permiten a la persona con Síndrome de Down efectuar una higiene dental de forma más completa, teniendo como mediador a sus profesores y padres para la adquisición de nuevos saberes (13).

Por lo tanto es responsabilidad del odontólogo mantener a los pacientes libres de caries, de periodontopatías; y mejorar las condiciones bucales de aquellos que ya presentan la enfermedad.

El objetivo de este estudio fue evaluar la aplicación de juegos colectivos adaptados a niños y adolescentes 
con Síndrome de Down, como estrategia de intervención educativa en la Salud Bucal.

\section{MATERIAL Y METODOS}

Se realizó un estudio de tipo observacional, prospectivo, longitudinal y comparativo. La muestra estuvo conformada por 50 pacientes con Síndrome de Down, estudiantes del Instituto de Rehabilitación infantil y Educación especial "Milagroso Niño Jesús" cuyo rango de edad fue de 6 a 32 años. Se diseñó e implemento un programa de actividades educativas en la que todos los pacientes participaron en todos los juegos colectivos; conforme llegaban a su cita se elaborada una historia clínica y se le realizaba un examen extraoral e intraoral; además, de la evaluación del índice de higiene oral según O’Leary (14).

Antes de iniciar con la sesión odontológica, se confeccionó una cartilla con los siguientes datos: Identifica el cepillo dental, identifica la pasta dental, identifica el hilo dental, identifica el enjuague bucal, sabe la técnica de cepillado, usa cepillo dental, cuantas veces se cepilla, sabe cuánta cantidad de pasta dental se coloca en el cepillo, usa cepillo dental, saber cómo usar el hilo dental, usa el enjuague bucal y cuantas veces usa el enjuague bucal, las preguntas relacionados con la salud bucal. Dicha cartilla fue llenada por el profesional de la salud en función de la experiencia y conocimientos de los pacientes asegurados. Luego, se les explicó en forma dinámica la técnica adecuada para realizar la higiene diaria, a cada uno de los pacientes (duración de 20 minutos cada sesión solo en fisioterapia).
La estrategia educativa, contenía los juegos que estaban orientados a incorporar los conocimientos en torno a la salud bucal; estos juegos se desarrollaron teniendo en cuenta el 1) Componente didáctico como herramienta de aprendizaje, que cumplía los siguientes requisitos: ser interesante, colectivo, permitir la interacción, respetar ritmos individuales, ser verificable y atractivo; 2) El ambiente y 3) La correlación entre patología, higiene y edad (atención, iniciativa, memoria a corto plazo y procesamiento de la información, memoria a largo plazo, correlación y análisis, operaciones secuenciales, operaciones numéricas). Estos juegos fueron elaborados con materiales de oficina y de decoración; los cuales se nombraron de la siguiente manera: Ruleta dental, memoria dental, armando la sonrisa y mi amiga la boca; orientados a incorporar conocimientos y a mejorar habilidades motoras, los cuales fueron aplicados durante 1 mes.

Como evaluación los pacientes niños, adolescentes y adultos de Essalud - Zarumilla entusiasmados me explicaban como debían de realizar su higiene diaria, como cepillarse, como usar el hilo dental y en qué casos usar enjuague bucal. Finalmente, se realizó el índice de higiene oral según O’Leary como indicador de mejoría en la higiene oral; y el llenado de la ficha de recolección de datos (14).

\section{RESULTADOS}

En el presente estudio se evaluó el uso de los juegos colectivos como una estrategia de intervención educativa en la salud bucal pacientes con Síndrome de

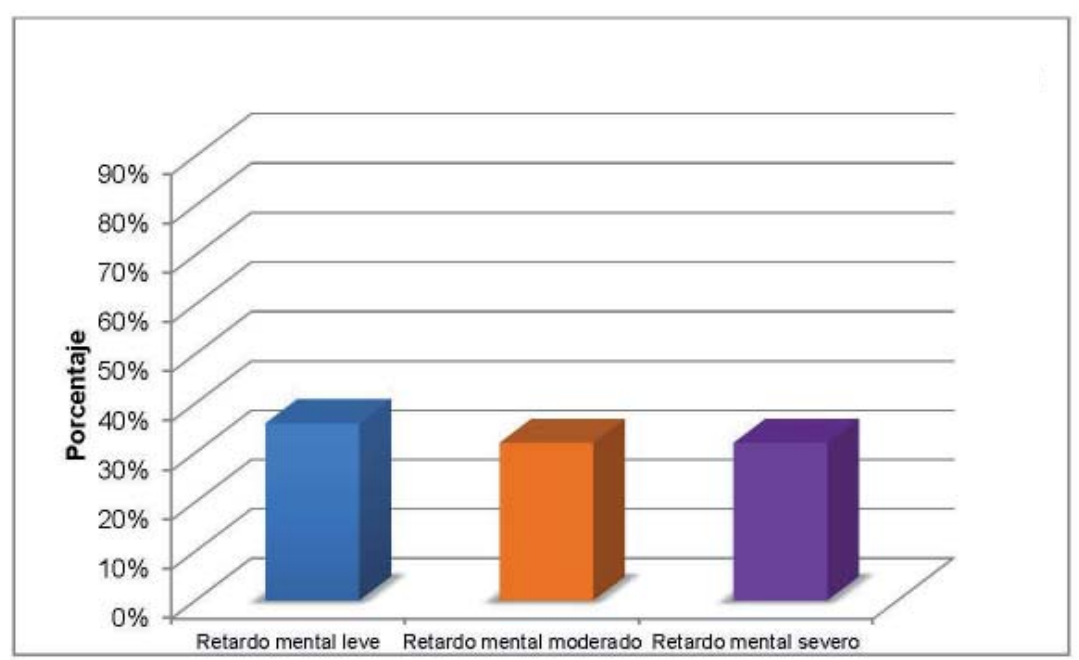

Grafico 1. Distribución del retardo mental, según severidad. 
Down del Instituto de rehabilitación infantil y educación especial “Milagroso niño Jesús”. Se evaluó a 50 estudiantes de los cuales el $58 \%$ pertenecieron al sexo masculino y el $42 \%$ al sexo femenino, siendo la edad promedio de los participantes de $14.76 \pm 5,99$ años, siendo la edad mínima 6 años y la edad máxima 32 años.

La distribución de retardo mental, según severidad nos muestra que el 36\% de los pacientes con Sindrome de Down tuvieron un retardo mental leve, 32\% un retardo mental moderado y el 32\% un retardo mental severo (Grafico 1).

Tras la aplicación de los juegos colectivos, se observa que el $98 \%$ ubica la boca donde corresponde; y el 2\% no la ubica, el 98\% identifica el cepillo dental; y el $2 \%$ no lo identifica, el 98\% identifica la pasta dental; y el 2\% no la identifica, el 96\% identifica el enjuague bucal; y el 4\% no lo identifica, el 98\% identifica el hilo dental; y el 2\% no lo identifica, el 98\% identifica el vaso con agua; y el $2 \%$ no lo identifica, el $98 \%$ identifica las partes que corresponden a la boca; y el $2 \%$ no las identifica, el $96 \%$ reconoce las formas de los dientes; y el $4 \%$ no reconoce las formas, el $94 \%$ sabe la técnica de cepillado; y el 6\% no sabe la técnica, el 96\% usa cepillo dental; y el 4\% no usa, el 92\% se cepillan 3 veces al día ; y el $8 \%$ no se cepillan, el $94 \%$ sabe que cantidad de pasta dental se coloca en el cepillo; y el $6 \%$ no sabe cuánta cantidad de pasta se coloca, el 98\% usa hilo dental; y el $2 \%$ no lo usa, el 96\% sabe cómo usar el hilo dental; y el 4\% no sabe cómo usar, el 94\% usa el enjuague bucal; y el 6\% no lo usa y el 94\% usa una vez el enjuague bucal; y el $6 \%$ no lo usa (Tabla 1).

Antes de la aplicación de los juegos colectivos, se observa que el índice de higiene oral, según la severidad del retardo mental; para los niños con retardo mental leve tuvo un valor del 71,42\%; los pacientes con retardo mental moderado tuvo un valor del $71,23 \%$ y por último los niños con Retardo mental severo tuvo un valor del 69,48\% (Tabla 2).

Los resultados muestran que el índice de higiene oral antes de la aplicación de la intervención educativa fue $70,78 \% \pm 9,6$; sin embargo, después de la intervención educativa el índice de higiene oral fue 66,60 $\pm 11,52$ demostrando que existe una diferencia significativa entre las medias del índice de higiene oral an- tes y después de la intervención educativa (Tabla 3). El promedio de índice de higiene oral obtenido antes

Tabla 1. Distribución de componentes de la Salud Oral que reconoce posterior a la implementación de la intervención educativa.

\begin{tabular}{|c|c|c|}
\hline & SI & NO \\
\hline Componentes de la Salud Oral & $(\%)(f)$ & $(\%)(f)$ \\
\hline Ubica la boca donde corresponde & $98(49)$ & $2(1)$ \\
\hline Identifica el cepillo dental & 98 (49) & $2(1)$ \\
\hline Identifica la pasta dental & $98(49)$ & $2(1)$ \\
\hline Identifica el enjuague bucal & $96(48)$ & $4(2)$ \\
\hline Identifica el hilo dental & 98 (49) & $2(1)$ \\
\hline Identifica el vaso con agua & 98 (49) & $2(1)$ \\
\hline $\begin{array}{l}\text { Identifica las partes que correspond } \\
\text { a la boca }\end{array}$ & 98 (49) & $2(1)$ \\
\hline Reconoce las formas de los dientes & $96(48)$ & $4(2)$ \\
\hline Sabe la técnica d cepillado & $94(47)$ & $6(3)$ \\
\hline Usa cepillo dental & $96(48)$ & $4(2)$ \\
\hline Cuantas veces se cepilla & $92(46)$ & $8(4)$ \\
\hline Sabe cuánta cantidad de pasta dental & & \\
\hline coloca en el cepillo & $94(47)$ & $6(3)$ \\
\hline usa el hilo dental & 98 (49) & $2(1)$ \\
\hline Saber cómo usar el hilo dental & $96(48)$ & $4(2)$ \\
\hline Usa el enjuague bucal & $94(47)$ & $6(3)$ \\
\hline Cuantas veces usa el enjuague bucal & $94(47)$ & $6(3)$ \\
\hline
\end{tabular}

Tabla 2. Índice de higiene oral, antes y después de la aplicación de los juegos colectivos, según severidad de retardo mental.

\begin{tabular}{lll}
\hline Retardo Mental & IHO Antes & IHO Después \\
\hline Leve & 71,42 & 67,56 \\
Moderado & 71,23 & 66,83 \\
Severo & 69,48 & 65,22 \\
\hline
\end{tabular}

Tabla 3. Comparación del Índice de Higiene Oral en los pacientes con Síndrome de Down, antes y después de la aplicación de la intervención educativa.

\begin{tabular}{lcccccc}
\hline & & Antes & \multicolumn{3}{c}{ Después } & \\
& $\mathrm{n}$ & media & DS & media & DS & $p$-value \\
\hline $\begin{array}{l}\text { Índice de } \\
\text { Higiene Oral }\end{array}$ & 50 & 70,78 & 9,6 & 66,60 & 11,52 & 0,000 \\
\hline
\end{tabular}

DS: Desviación estándar; Prueba t para muestras relacionadas $(\mathrm{p}<0,05)$ 
de la intervención educativa en el retardo mental leve fue $71,42 \%$ y después fue $67,56 \%$; hay diferencia significativa entre las medias del IHO de los pacientes con retardo mental leve antes y después de la intervención $(\mathrm{p}<0,05)$. Se observó que en el promedio de índice de higiene oral obtenido antes de la intervención educativa en el retardo moderado fue $71,23 \%$ y después fue $66,83 \%$; hay diferencia significativa entre las medias del IHO de los niños y adolescente con retardo mental moderado antes y después de la intervención $(p<0,05)$. Se observó que en el promedio de índice de higiene oral obtenido antes de la intervención educativa en el retardo moderado severa fue $69,54 \%$ y después fue $65,22 \%$; hay diferencia significativa entre las medias del IHO de los pacientes con retardo mental severo antes y después de la intervención $(\mathrm{p}<0,05)$ (Tabla 4). ticipantes mostraron que 98\% ubican la boca donde corresponde, 98\% identifican el cepillo dental, 98\% identifican la pasta dental, 96\% identifican el enjuague bucal, 98\% identifican el hilo dental, 98\% identifican el vaso con agua, 98\% identifican las partes que corresponden a la boca, 96\% reconoce las formas de los dientes; 94\% sabe la técnica d cepillado; 96\% usa cepillo dental; 92\% se cepillan 3 veces al día; 94\% sabe cuánta cantidad de pasta dental se coloca en el cepillo; 98\% usa el hilo dental; 96\% saben cómo usar el hilo dental; 94\% usa el enjuague bucal y 94\% usa una vez el enjuague bucal. Estos resultados se complementaron con la comparación del Índice de higiene oral antes de la intervención que fue 70,78\% \pm 9 ,6; y después de la intervención $66,60 \% \pm 11,52$; encontrándose diferencia estadísticamente significativa entre las variables $(\mathrm{p}<0,05)$.

Tabla 4. Comparación del Îndice de Higiene Oral (IHO) en los pacientes con Síndrome de Down según severidad de la enfermedad, antes y después de la aplicación de los juegos colectivos.

\begin{tabular}{llllll}
\hline & \multicolumn{2}{l}{ IHO Antes } & \multicolumn{2}{l}{ IHO Después } \\
Retardo Mental & media & DS & media & DS & p \\
\hline Leve & 71,42 & 9,80 & 67,56 & 11,03 & 0,001 \\
Moderado & 71,23 & 9,10 & 66,83 & 10,31 & 0,000 \\
Severo & 69,54 & 10,60 & 65,22 & 13,78 & 0,001 \\
\hline
\end{tabular}

DS: Desviación estándar; Prueba t para muestras relacionadas ( $<<0,05)$.

\section{DISCUSIÓN}

Los resultados muestran que hubo una mejoría en el nivel de conocimiento de los niños y adolescentes con Síndrome de Down, lo cual se vio reflejado en los datos obtenidos en la investigación y en el índice de higiene oral tomado antes y después de la intervención educativa $(\mathrm{p}<0,05)$ con juegos colectivos diseñados para este estudio. en cuanto a la muestra analizada, los pacientes de 6 a 32 años del Instituto de Rehabilitación Infantil y Educación Especial presentaron en su mayoría retardo mental leve, seguido de retardo mental moderado y severo.

Para este estudio se elaboró distintos juegos con nombres, colores vivos, imágenes y formas llamativas; posteriormente se pasó a la implementación. Después de la intervención educativa se pasó a la fase de evaluación basándose en la identificación de los componentes de la salud oral, la mayoría de los par-
Aunque no existen antecedentes de estudios parecidos a éste, no se pueden comparar los resultados con los estudios previos ya que la metodología y muestra estudiada difieren; sin embargo, la aplicación de estrategias educativas para ampliar los conocimientos en Salud Oral, han resultado eficaces. Moncunill et al., realizaron una investigación que tuvo como objetivo diseñar, implementar y evaluar juegos colectivos con componente en personas con síndrome de Down, como estrategias mediadoras para la promoción de la salud bucal, obteniendo que los juegos con contenidos de salud bucal dotaron a los mediadores de una herramienta apropiada para que los niños y adolescentes lograran la apropiación de conductas saludables (14). Mejía evaluó la relación entre el conocimiento sobre higiene bucal de padres y el estado de higiene bucal de personas con habilidad diferente (16); al relacionar el conocimiento de higiene bucal de padres, con la higiene bucal del paciente con habilidad diferente, se observó que el $84,21 \%$ de los pacientes cuyos padres 
poseían un conocimiento bueno obtuvieron una buena higiene bucal; de esta manera también se encontró que el $100 \%$ de los pacientes cuyos padres presentaron un conocimiento malo no presentaron buena higiene bucal. Pinto evaluó el impacto de un programa preventivo, concluyendo que la intervención educativa -lúdica produce un aumento en el nivel de conocimientos de salud bucal en pacientes escolares con deficiencia auditiva (14).

Araujo llevó una propuesta de un programa preventivo promocional a los alumnos con retardo mental y con síndrome de Down, observó al examen oral deficiente higiene oral y caries dental (17). La propuesta para modificar ciertos hallazgos se basó en realizar un programa preventivo que era el manual. Se motivó a los padres y maestros mostrándoles el estado actual de higiene oral de los alumnos, esto ayudó a la motivación; quienes brindaron su apoyo y se comprometieron a supervisar el programa. Es importante realizar ciertas técnicas como decir, mostrar y hacer, reforzamiento positivo, modelos, y sobre todo la desensibilización; por lo mismo que los participantes son únicos; los gustos, alegrías son especiales, a fin de mostrarles seguridad, confianza y confort.

\section{CONCLUSIONES}

Se concluye que, con la aplicación de la estrategia educativa; se logró una disminución del índice de higiene oral; asimismo, se obtuvo un aprendizaje de los componentes de la salud oral en los tres grupos de retardo mental; con una mejoraría significativa posterior a la implementación de la estrategia.

\section{Correspondencia:}

Jackelym Aguirre Córdova

Correo electrónico: jackelym@hotmail.com

\section{REFERENCIAS BIBLIOGRAFICAS}

1. Rondal J. Educar y hacer hablar al niño Down. 1ra Edición. Mexico DF: Editorial Trillas; 1995

2. Rubin E, Farber J. Patología. México DF: Editorial Panamericana S.A;1990

3. Jimenez MT, Paulino D, Martin JM. La clasificación internacional del funcionamiento de la discapacidad y de la salud (CIF) 2001. Rev Esp Salud Publica. 2002; 76(4): 271-279.

4. Saez U, Castejon I, Molina A, Roncero M. Estudio epidemiológico dela salud bucodental en pacientes conpa-
rálisiscerebral.RevClinMedFam.2008;2(5):206-209.

5. Mouradian WE, Corbin SB. Addressing health disparities through dental-medical collaborations, Part II. Cross-cutting themes in the care of special populations. J Dent Educ. 2003; 67(12): 1320-1326.

6. Waldman H. Almost four million children with disabilities. Journal of dentistry for children. 1995;62(3): 205-209.

7. Kumar S, Sharma J, Duraiswamy P, Kulkarni S. Determinates for oral hygiene and periodontal status among mentally disabled children and adolescents. J Indian Soc Pedod Prevent Dent. 2009; 27(3): 151-157.

8. Morales MC. Patologías bucodentales y alteraciones asociadas prevalentes en una población depacientes con parálisis cerebral. Acta Odont Venez. 2008; 46(1):1-7.

9. Glassman P, Anderson M, Jacobsen P, Schonfeld S, Weintraub J, White A. Practical protocols for the prevention of dental disease in community settings for people with special needs. Spec Care Dentist. 2003; 23(5): 160-164.

10. Reuland-Bosma W, Van der Reijden WA, Van Winkelhoff AJ. Absence of a specific subgingival microflora in adults with Down's syndrome. J Clin Periodontol. 2001; 28(11): 1004-1009.

11. Demicheri R, Battle A. La enfermedad periodontal asociada al paciente con Síndrome de Down. Odontoestomatología. 2011; 13 (18): 4-15.

12. Cumella S, Ransford N, Lyons J, Burnham H. Needs for oral care among people with intellectual disability not in contact with Community Dental Services. J Intellect Disabil Res. 2000; 44: 45-52.

13. Chavarría MG. La persona con necesidades especiales y su salud oral. Odontología Vital. 2011; 15: 30-39.

14. Pinto $\mathrm{H}$. Estrategias de intervención educativa en odontología dirigida a escolares con deficiencia auditiva. Tesis para obtener el título profesional de cirujano dentista. Lima, Perú: Facultad de Odontología, Universidad Nacional Mayor de San Marcos; 2009.

15. Moncunill E, Calamri G, Cornejo L. Estrategia mediadora para la promoción de salud bucal en niños y adolescentes con síndrome de Down. Revista Síndrome de Down. 2007; 24: 62-7.

16. Mejía M. Conocimiento de higiene bucal de padres relacionado con la higiene bucal de niños con habilidades diferentes.Tesis para obtener el título profesional de cirujano dentista. Lima, Perú: Facultad de Odontología, Universidad Nacional Federico Villarreal; 2009.

17. Araujo J. Propuesta de un programa preventivo promocional de salud bucal en alumnos discapacitados. Callao, Perú: Centro de educación especial Santa Teresa de Couderc; 1997

Recibido : 24/03/2015

Aceptado: 17/05/2015 